$1-1-2008$

\title{
Defects and symmetry influence on visible emission of Eu doped nanoceria
}

\author{
Suresh Babu \\ University of Central Florida \\ Alfons Schulte \\ University of Central Florida \\ Sudipta Seal \\ University of Central Florida
}

Find similar works at: https://stars.library.ucf.edu/facultybib2000 University of Central Florida Libraries http://library.ucf.edu

This Article is brought to you for free and open access by the Faculty Bibliography at STARS. It has been accepted for inclusion in Faculty Bibliography 2000 s by an authorized administrator of STARS. For more information, please contactSTARS@ucf.edu.

\section{Recommended Citation}

Babu, Suresh; Schulte, Alfons; and Seal, Sudipta, "Defects and symmetry influence on visible emission of Eu doped nanoceria" (2008). Faculty Bibliography 2000s. 90.

https://stars.library.ucf.edu/facultybib2000/90

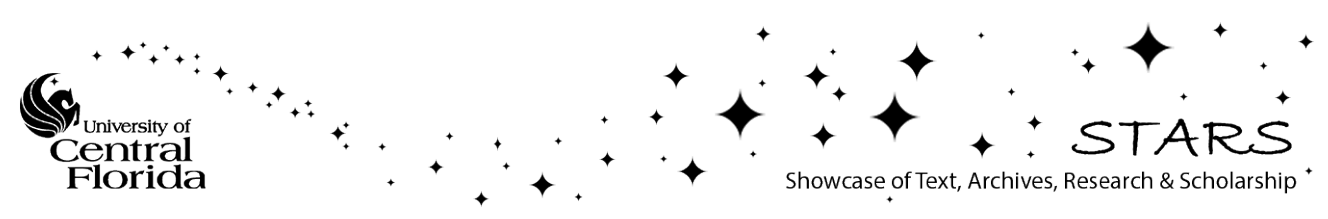




\section{Defects and symmetry influence on visible emission of Eu doped nanoceria}

Cite as: Appl. Phys. Lett. 92, 123112 (2008); https://doi.org/10.1063/1.2904627

Submitted: 19 January 2008 . Accepted: 10 March 2008. Published Online: 28 March 2008

Suresh Babu, Alfons Schulte, and Sudipta Seal

\section{ARTICLES YOU MAY BE INTERESTED IN}

Size dependency variation in lattice parameter and valency states in nanocrystalline cerium oxide

Applied Physics Letters 87, 133113 (2005); https://doi.org/10.1063/1.2061873

Cerium oxide nanoparticles: Size-selective formation and structure analysis

Applied Physics Letters 80, 127 (2002); https://doi.org/10.1063/1.1430502

Role of trivalent La and Nd dopants in lattice distortion and oxygen vacancy generation in cerium oxide nanoparticles

Applied Physics Letters 88, 243110 (2006); https://doi.org/10.1063/1.2210795

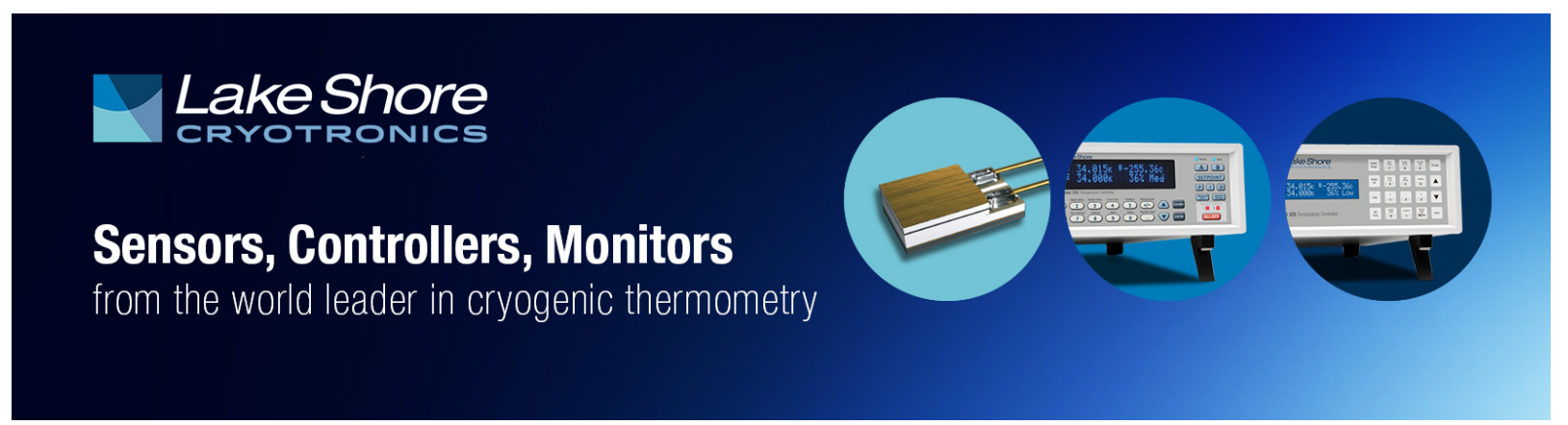




\title{
Defects and symmetry influence on visible emission of Eu doped nanoceria
}

\author{
Suresh Babu, ${ }^{1}$ Alfons Schulte, ${ }^{2}$ and Sudipta Seal ${ }^{1, a)}$ \\ ${ }_{1}^{1}$ Advanced Materials Processing and Analysis Centre (AMPAC), Nanoscience Technology Center \\ and Mechanical, Materials and Aerospace Engineering (MMAE), University of Central Florida, \\ 4000 Central Florida Boulevard, Orlando, Florida 32816, USA \\ ${ }^{2}$ Department of Physics, University of Central Florida, 4000 Central Florida Boulevard, Orlando, \\ Florida 32816, USA
}

(Received 19 January 2008; accepted 10 March 2008; published online 28 March 2008)

\begin{abstract}
Europium doped cerium oxide particles of $10 \mathrm{~nm}$ were synthesized by room temperature chemical precipitation technique and annealed at 500 and $900{ }^{\circ} \mathrm{C}$ to study its effect on luminescence. X-ray photoelectron spectroscopic result shows an increase in $\mathrm{Ce}^{3+}$ concentration from $20 \%$ to $23 \%$ on Eu doping but decreases to $8 \%$ on annealing. Raman studies show a progressive blueshift from 461 to $464 \mathrm{~cm}^{-1}$ due to local symmetry ordering with temperature. Emission intensity varies with the wavelength of excitation and observed transitions indicate the presence of $\mathrm{Eu}^{3+}$ in different symmetry environments. (C) 2008 American Institute of Physics. [DOI: 10.1063/1.2904627]
\end{abstract}

Nanostructures of rare-earth cerium oxide or ceria $\left(\mathrm{CeO}_{2}\right)$ have a wide range of applications such as electrolyte in solid oxide fuel cell, oxygen gas sensors, chemical polishing, and catalyst. Recently, beneficial therapeutic properties of ceria nanoparticles have been reported. ${ }^{1}$ Since ceria exhibits weak luminescence, doping with rare earths such as europium $(\mathrm{Eu})$ can enhance the visible emission required for imaging. Although emission properties of Eu doped in various matrices have been studied, 2,3 only a few have been attempted in ceria. Synthesis of Eu doped in ceria by sol-gel ${ }^{4}$ and high temperature reaction ${ }^{5}$ have been reported. However, characteristic $\mathrm{Eu}^{3+}$ emission was not observed for $3.5 \mathrm{~nm}$ nanocrystals prepared by nonhydrolytic solution route. ${ }^{6}$ In the present communication, we report a simple room temperature technique for the synthesis of Eu doped ceria nanoparticles and correlate the influence of surface and structural symmetries on luminescence characteristics as a function of annealing temperature.

Europium doped (5 wt \%) nanoceria was synthesized from the aqueous cerium nitrate hexahydrate (Aldrich, 99\%) and europium nitrate trihydrate (Aldrich, 99\%) solutions by hydrolysis with ammonia. Resultant powders were washed with water and dried at $100{ }^{\circ} \mathrm{C}$ overnight. Similar procedure was used for preparing ceria nanoparticles (C). Eu doped ceria nanoparicles were coded as CE, CE-500, and CE-900 for Eu doped ceria, 500 and $900{ }^{\circ} \mathrm{C}$ heat treated samples, respectively (Table I). The powders were characterized using high resolution transmission electron microscopy (HRTEM), X-ray diffraction (XRD), x-ray photoelectron spectroscopy (XPS), Raman, and infrared (IR) spectroscopy.

The HRTEM images were obtained with Philips Tecnai F30 at an operational voltage of $300 \mathrm{keV}$. The bright field HRTEM images of C, CE, and CE-500 shown in Fig. 1 exhibit nearly spherical 7-13 nm particles, with lattice fringes corresponding to the stable (111) plane. However, CE-900 consists of agglomerate with a mean particle size of $37 \mathrm{~nm}$. On annealing to $900{ }^{\circ} \mathrm{C}$, individual nanoparticles come in

\footnotetext{
${ }^{a)}$ Electronic mail: sseal@mail.ucf.edu.
}

contact and align to form octahedral shape in order to minimize the interfacial energy. ${ }^{7}$

The crystal structure was determined with XRD (Rigaku) using $\mathrm{Cu} \mathrm{K} \alpha$ radiation. Figure 2(a) shows the XRD pattern for nanoparticles. Reflections can be indexed to fluorite structure of $\mathrm{CeO}_{2}$ (Ref. 8). The lattice parameter of $\mathrm{C}$ was found to be $0.5418 \mathrm{~nm}$, higher than that reported for bulk ceria $(0.541 \mathrm{~nm})$. Due to the difference in ionic radii between $\mathrm{Ce}^{3+}(0.1283 \mathrm{~nm})$ and $\mathrm{Ce}^{4+}(0.1098 \mathrm{~nm})$, the lattice parameter of $\mathrm{C}$ is higher than that of bulk ceria. ${ }^{9}$ Since Eu has higher ionic radii $\left(0.121\right.$ and $0.126 \mathrm{~nm}$ for $\mathrm{Eu}^{3+}$ and $\mathrm{Eu}^{2+}$, respectively) than $\mathrm{Ce}^{4+}$, doping of $\mathrm{Eu}(\mathrm{CE})$ increases the lattice parameter to $0.5422 \mathrm{~nm}$, which decreases upon annealing. ${ }^{8}$ The observed broadness in the XRD peaks can be influenced by size as well as strain of the particles. Williamson-Hall plots were used to separate the effects of size and strain in the nanoparticles using

$$
\beta=\beta_{\text {size }}+\beta_{\text {strain }}=\frac{0.9 \lambda}{t \cos \theta}+\frac{4(\Delta d) \sin \theta}{d \cos \theta},
$$

where $\beta$ is the full width half maximum of the diffraction peaks after correcting for instrumental broadening, $\lambda$ is the wavelength of the incident $\mathrm{x}$ ray, $\theta$ is the diffraction angle, $t$ is the crystal size, and $\Delta d$ is the difference of the $d$ spacing corresponding to a typical peak. After correcting the instrumental broadening, a plot of $\beta \cos \theta$ against $4 \sin \theta$ yields the crystal size from the intercept value and the strain $(\Delta d / d)$ from the slope. With annealing temperature, mean crystallite size increases to $39.2 \mathrm{~nm}$ at $900{ }^{\circ} \mathrm{C}$ with a corresponding

TABLE I. Size, strain and $\mathrm{Ce}^{3+}$ concentration for nanoparticles.

\begin{tabular}{|c|c|c|c|c|c|c|}
\hline Sample & $\begin{array}{l}\mathrm{Wt} \% \\
\text { of } \mathrm{Eu}\end{array}$ & $\begin{array}{c}\text { Annealing } \\
\text { temperature } \\
\left({ }^{\circ} \mathrm{C}\right)\end{array}$ & $\begin{array}{l}\text { Size } \\
(\mathrm{nm})\end{array}$ & Strain & $\begin{array}{l}\text { Lattice } \\
\text { parameter } \\
\quad(\mathrm{nm})\end{array}$ & $\begin{array}{c}\mathrm{Ce}^{3+} \\
\text { concentration } \\
(\%)\end{array}$ \\
\hline $\mathrm{C}$ & $\cdots$ & $\cdots$ & 9.6 & $8.5 \times 10^{-3}$ & 0.5418 & 20 \\
\hline $\mathrm{CE}$ & 5 & $\cdots$ & 10.1 & $9.9 \times 10^{-3}$ & 0.5422 & 23 \\
\hline CE-500 & 5 & 500 & 12.9 & $5.3 \times 10^{-3}$ & 0.5413 & 13 \\
\hline CE-900 & 5 & 900 & 39.2 & $7.9 \times 10^{-4}$ & 0.5412 & 8 \\
\hline
\end{tabular}



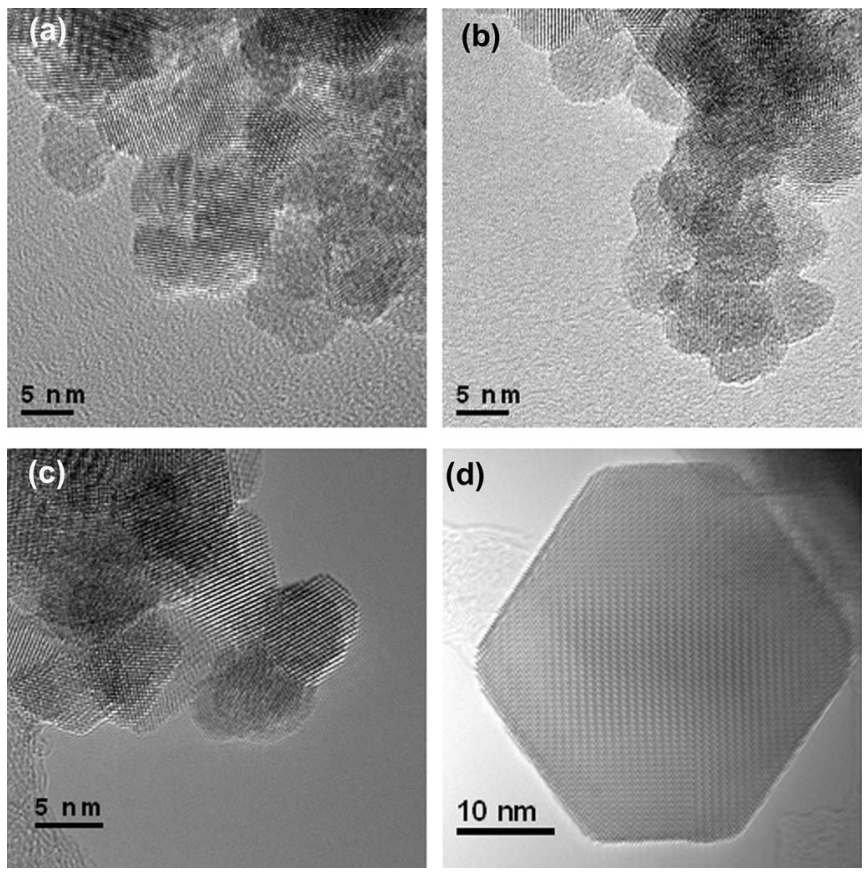

FIG. 1. HRTEM images of (a) C, (b) CE, (c) CE-500, and (d) CE-900.

decrease in strain due to lattice ordering (Table I).

In order to identify the oxidation state of $\mathrm{Ce}$ and $\mathrm{Eu}$, XPS study was carried out with Perkin-Elmer (PHI 5400 ESCA) spectrometer. Figure 2(b) shows the recorded spectra for nanoparticles and deconvoluted peaks were used for calculating the concentration of $\mathrm{Ce}^{3+}$ (Table I), as reported earlier. ${ }^{\mathrm{T}, 11}$ The concentration of $\mathrm{Ce}^{3+}$ increases on doping with $\mathrm{Eu}^{3+}$ but decreases on annealing due to the conversion of $\mathrm{Ce}^{3+}$ to $\mathrm{Ce}^{4+}$. The inset of Fig. 2(b) shows XPS spectra for Eu $3 d$ showing two characteristic peaks at 1134 and $1163 \mathrm{eV}$ corresponding to +3 state and the absence of low binding energy peaks suggest that $\mathrm{Eu}$ is present in the +3 state. $^{12}$

Raman scattering is a useful technique to detect oxygen sublattice distortions as electron-phonon (lattice vibration) interactions are very sensitive to local environments. Raman spectroscopic studies were carried out with a Horbia Jobin Yvon LabRam IR micro-Raman system with a He-Ne laser at $632.8 \mathrm{~nm}$. Bulk ceria among the Raman allowed modes exhibits a symmetric, sharp peak centered at $465 \mathrm{~cm}^{-1}$ corresponding to a breathing (phonon) mode of $\mathrm{O}^{2-}$ anions around $\mathrm{Ce}^{4+}$ cation, which are sensitive to any disorder in the oxygen sublattice resulting from nonstoichiometry. ${ }^{13}$ Figure 2(c) shows the Raman spectra for all samples. Ceria nanoparticle (C) shows a broad asymmetric peak at a lower frequency of $461 \mathrm{~cm}^{-1}$ than bulk ceria due to smaller particle size, which generates larger defect concentration. ${ }^{14}$ On annealing, peaks get sharper and symmetric, with a progressive shift in the position from 461 (CE), 462 (CE-500), and $464 \mathrm{~cm}^{-1}$ (CE-900). The decrease in the asymmetry is attributed to the improved phonon lifetime due to the growth in nanocrystal size. ${ }^{13}$ Annealing reduces the structural gradient induced by the surface strain of nanoparticles. Oxygen vacancies from the bulk of the particle become unstable and tend to migrate to the surface and annihilate, as shown by a decrease in $\mathrm{Ce}^{3+}$ concentration in the XPS results. To understand the role of surface chemical modifications, IR spectra were recorded using Perkin-Elmer Spectrum one. IR spectra of ceria and Eu doped ceria samples exhibit a broad peak centered around $3400 \mathrm{~cm}^{-1}$ correspond to the $\mathrm{O}-\mathrm{H}$ symmetric stretching from the surface hydroxyl group due to basic conditions used [Fig. 2(d)] and the reaction can be represented as

$$
\begin{aligned}
& \mathrm{Ce}^{3+}+3 \mathrm{OH}^{-} \rightarrow \mathrm{Ce}(\mathrm{OH})_{3}, \\
& \mathrm{Ce}(\mathrm{OH})_{3}+\mathrm{H}_{2} \mathrm{O} \rightarrow \mathrm{Ce}(\mathrm{OH})_{4}+\mathrm{H}^{+}+\mathrm{e}^{-}, \\
& \mathrm{Ce}(\mathrm{OH})_{4} \rightarrow \mathrm{CeO}_{2}+2 \mathrm{H}_{2} \mathrm{O} .
\end{aligned}
$$

As a result of surface dehydroxylation on annealing, $\mathrm{OH}$ group intensity decreases for CE-500 and CE-900.

To evaluate the emission characteristics, luminescence measurement were carried out using Hitachi-7000 spectro-
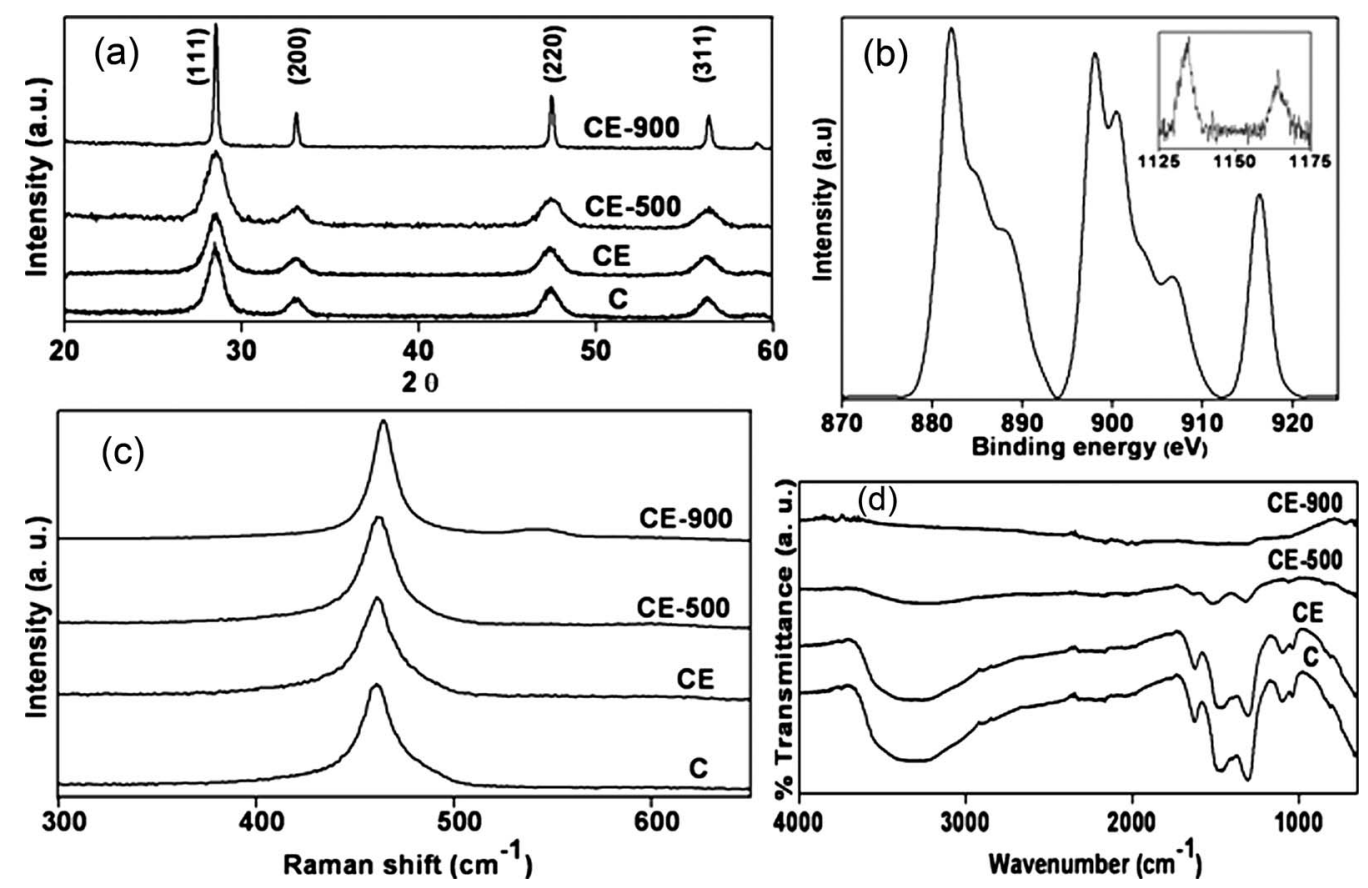

FIG. 2. (a) XRD spectra of nanoparticles. (b) Representative Ce and Eu (inset) $3 d$ level XPS spectra of CE. (c) Raman and (d) IR spectra of nanoparticles. 

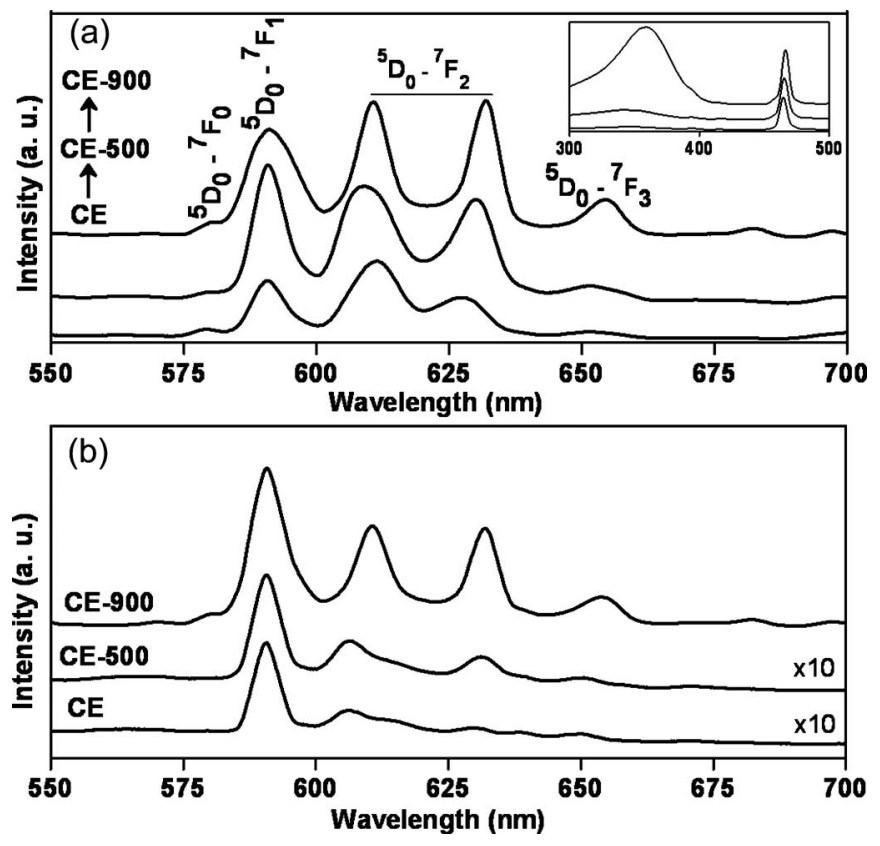

FIG. 3. Emission spectra of nanoparticles excited at 466 (a) and $370 \mathrm{~nm}$ (b). The inset shows excitation spectra $\left(\lambda_{\mathrm{em}}=611 \mathrm{~nm}\right)$.

photometer. The inset of Fig. 3(a) shows excitation spectra followed at emission wavelength of $611 \mathrm{~nm}$. The sharp peak at $466 \mathrm{~nm}$ corresponds to Eu $4 f-4 f$ transition, and the broad peak results from the overlap between $\mathrm{Ce}^{4+}-\mathrm{O}^{2-}$ charge transfer and intraconfigurational Eu $4 f-4 f$ transitions. Figure $3(\mathrm{a})$ shows the characteristic $\mathrm{Eu}^{3+}$ emission ${ }^{5} D_{0^{-}}{ }^{7} F_{J}$ $(J=0,1,2$, etc. $)$ for nanoparticles excited at $466 \mathrm{~nm}$. The emission corresponds to ${ }^{5} D_{0^{-}}{ }^{7} F_{0} \quad(579 \mathrm{~nm}), \quad{ }^{5} D_{0^{-}}{ }^{7} F_{1}$ $(591 \mathrm{~nm}),{ }^{5} D_{0^{-}}{ }^{7} F_{2}(611$ and $632 \mathrm{~nm})$, and ${ }^{5} D_{0^{-}}{ }^{7} F_{3}(654 \mathrm{~nm})$ transitions. The ${ }^{5} D_{0^{-}}{ }^{7} F_{1}$ lines originate from the magnetic dipole (MD) transition, while ${ }^{5} D_{0^{-}}{ }^{7} F_{2}$ lines originate from electric dipole (ED) transition. According to the Judd-Ofelt theory, ED transition is only allowed in the absence of inversion symmetry, and is sensitive to the local electric field. ${ }^{3}$ $\mathrm{CeO}_{2}$ has a fluorite structure with $\mathrm{Ce}$ located in octahedral symmetry. When $\mathrm{Eu}^{3+}$ ions locate at sites with inversion symmetry, MD transition occurs; otherwise, ED transition dominates. The splitting of electric field sensitive ${ }^{5} D_{0}-{ }^{7} F_{2}$ transition indicates that $\mathrm{Eu}^{3+}$ exists in at least two structural environments differing in symmetry. The low intensity of ${ }^{5} D_{0}{ }^{-} F_{0}$ line is due to the fact that this transition is forbidden by both ED and MD selection rules.

The emission mechanism of rare earth-doped materials is generally through energy transfer from the excited host to the dopant and/or direct charge carrier trapping by the dopant molecule. Emission spectrum of nanoparticles excited near the band edge of ceria (370 nm) (Ref. 15) is shown in Fig. 3(b). The emission spectra show strong $591 \mathrm{~nm}$ peak while direct $\mathrm{Eu}^{3+}$ excitation at $466 \mathrm{~nm}$ shows less intense peak at $591 \mathrm{~nm}$ than at 611 and $632 \mathrm{~nm}$, indicating the difference in the energy transfer mechanism. On excitation at $370 \mathrm{~nm}$, en- ergy transfer from host to $\mathrm{Eu}^{3+}$ occurs, which becomes efficient on annealing. Li et al. ${ }^{16}$ correlated the electronegativity of rare earth on dehydroxylation with temperature, and Tsunekawa et al. ${ }^{11}$ observed that in the nanodomain, the surface of the particle predominantly has $\mathrm{OH}$ termination rather than $\mathrm{O}_{2}^{2-}$. Higher electronegativity of $\mathrm{Eu}^{3+}$ over $\mathrm{Ce}^{3+}$ leads to stronger binding with the surface hydroxyl group, resulting in higher $\mathrm{Eu}^{3+}$ concentration on the surface or subsurface. Hydroxyl groups provide an effective pathway for the radiationless energy transfer of $\mathrm{OH}$ vibration and quench the emission intensity in the as prepared conditions (CE). Annealing reduces $\mathrm{OH}$ groups, leading to an enhanced ${ }^{5} D_{0^{-}}{ }^{7} F_{2}$ emission. The ratio of ED to MD transition increases with temperature as a result of stronger $\mathrm{Eu}-\mathrm{O}$ covalent bond. Since Ce can exist in +3 and +4 oxidation states, coexistence of $\left(\mathrm{Ce}^{3+}-\mathrm{O}-\mathrm{Eu}^{3+}\right)$ and $\left(\mathrm{Ce}^{4+}-\mathrm{O}-\mathrm{Eu}^{3+}\right)$ arrangements in the crystalline environment is possible. On annealing, $\mathrm{Ce}^{4+}$ concentration increases; this leads to predominant $\left(\mathrm{Ce}^{4+}-\mathrm{O}-\mathrm{Eu}^{3+}\right)$ arrangement. Smaller positive charge makes $\mathrm{Ce}^{4+}$ more electronegative, leading to more covalent character of Eu-O bond resulting in enhanced ED transition.

In summary, Eu doped ceria nanoparticles were synthesized by room temperature wet chemical precipitation technique. Size of the particles increased from 10 to $39 \mathrm{~nm}$ on annealing with retained fluorite structure. The ratio of $\mathrm{Ce}^{4+}$ to $\mathrm{Ce}^{3+}$ is one of the important factors in determining the $\mathrm{Eu}^{3+}$ emission. A reduction in the surface hydroxyl, defect, and $\mathrm{Ce}^{3+}$ concentration results in enhanced emission intensity. Depending on the wavelength of excitation, emission pattern varies due to the difference in energy transfer mechanisms. The present result shows that the local chemical environments, as well as defects, have a strong influence on the luminescence properties of nano rare earth emitters.

This research (various sections) is funded by NSF NIRT CBET: 0708172, NSF CMII: 0629080, and NSF MRI: DMR-0421253.

${ }^{1}$ J. Chen, S. Patil, S. Seal, and J. F. McGinnis, Nat. Nanotechnol. 1, 142 (2006).

${ }^{2}$ P. Mohanty and S. Ram, J. Mater. Chem. 13, 3021 (2003).

${ }^{3}$ R. Reisfeld, E. Zigansky, and M. Gaft, Mol. Phys. 102, 1319 (2004).

${ }^{4}$ S. Fujihara and M. Oikawa, J. Appl. Phys. 95, 8002 (2004).

${ }^{5}$ X. Liu, S. Chen, and X. Wang, J. Lumin. 127, 650 (2007).

${ }^{6}$ Z. Wang, Z. Quen, and J. Lin, Inorg. Chem. 46, 5237 (2007).

${ }^{7}$ Z. L. Wang and X. Feng, J. Phys. Chem. B 107, 13563 (2003).

${ }^{8}$ JCPDS Card No. 810792.

${ }^{9}$ R. D. Shannon and C. T. Prewitt, Acta Crystallogr. B26, 1046 (1970).

${ }^{10}$ S. Deshpande, S. Patil, S. V. N. T. Kuchibhatla, and S. Seal, Appl. Phys. Lett. 87, 133113 (2006)

${ }^{11}$ S. Tsunekawa, T. Fukuda, and A. Kasuya, Surf. Sci. 457, L437 (2000).

${ }^{12}$ E. J. Cho and S. J. Oh, Phys. Rev. B 59, R15613 (1999).

${ }^{13}$ J. R. McBride, K. C. Hass, B. D. Poindexter, and W. H. Weber, J. Appl. Phys. 76, 2435 (1994).

${ }^{14}$ S. Patil, S. Seal, Y. Guo, A. Schulte, and J. Norwood, Appl. Phys. Lett. 88, 243110 (2006).

${ }^{15}$ S. Tsunekawa, T. Fukuda, and A. Kasuya, J. Appl. Phys. 87, 1318 (2000).

${ }^{16}$ K. Li and D. Xue, Phys. Status Solidi B 244, 1982 (2007). 